\section{Original Article}

Korean J Transplant 2020;34:100-108

https://doi.org/10.4285/kjt.2020.34.2.100

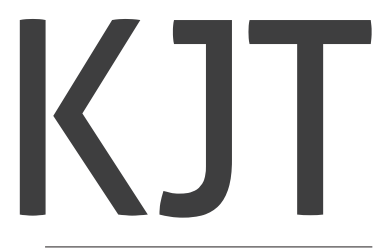

pISSN 2671-8790

elSSN 2671-8804

\title{
Hepatocyte and mesenchymal stem cell co- transplantation in rats with acute liver failure
}

\author{
Cheng-Maw Ho ${ }^{1,2,3}$, Ya-Hui Chen ${ }^{3}$, Chin-Sung Chien ${ }^{3}$, Shu-Li Ho ${ }^{1,2, *}$, \\ Hui-Ling Chen ${ }^{3}$, Rey-Heng Hu, ${ }^{1,2}$, Po-Huang Lee ${ }^{1,2,4}$ \\ ${ }^{1}$ Department of Surgery, National Taiwan University Hospital, Taipei, Taiwan \\ ${ }^{2}$ College of Medicine, National Taiwan University, Taipei, Taiwan \\ ${ }^{3}$ Hepatitis Research Center, National Taiwan University Hospital, Taipei, Taiwan \\ ${ }^{4}$ Department of Surgery, E-Da Hospital, I-Shou University, Kaohsiung, Taiwan
}

Received February 9, 2020

Revised March 26, 2020

Accepted March 27, 2020

Corresponding author: Hui-Ling Chen Hepatitis Research Center, National

Taiwan University Hospital, 7 ChungShan South Road, Taipei 100, Taiwan Tel: +886-2-2312-3456-67505

Fax: +886-2-2382-5962

E-mail: hlchen9@ntu.edu.tw

*Current affiliation: Department of

Anatomy and Cell Biology, National

Yang-Ming University, Taipei, Taiwan

(C) The Korean Society for Transplantation This is an Open Access article distributed under the terms of the Creative Commons Attribution Non-Commercial License (http://creativecommons.org/licenses/ by-nc/4.0/) which permits unrestricted non-commercial use, distribution, and reproduction in any medium, provided the original work is properly cited.

Background: Cell therapy is considered a potential alternative to liver transplantation in acute liver failure (ALF). We aimed to evaluate the add-on therapeutic benefit of hepatocyte and mesenchymal stem cell (MSC) cotransplantation over hepatocyte-only transplantations in a rat model of ALF.

Methods: ALF was induced by D-galactosamine in Sprague-Dawley rats. Freshly isolated donor hepatocytes were derived from $\mathrm{Tg}$ (UBC-emGFP) rats and MSCs were collected from the bone marrow cells of DsRed rats. Donor hepatocytes $\left(1 \times 10^{7} / \mathrm{mL}\right)$ were intraportally transplanted 24 hours after treatment with $\mathrm{D}$-galactosamine over a 70 -second interval, and donor MSCs $\left(0.5,1\right.$, or $\left.2 \times 10^{6} / 0.5 \mathrm{~mL}\right)$ were intraportally transplanted 1 hour after the hepatocyte transplantation was complete. Animals were sacrificed after 7 and 14 days and subjected to donor cell identification, liver histology, serologic testing, and immunohistopathological examination.

Results: MSCs were observed in the periportal area, 1 and 2 weeks after transplantation. Transplanted hepatocytes did not actively proliferate when compared to hepatocyte-only transplantation. Morphologically, transplanted MSCs did not appear to differentiate into hepatocytes even 2 weeks after transplantation. Cotransplantation of MSCs was associated with lower macrophage infiltration, and reduced type I collagen, hepatocyte growth factor, tumor necrosis factor- $\alpha$, and interleukin 10 expression, with similar gene expression profiles for epidermal growth factor and interleukin 6, when compared to hepatocyte-only transplantation.

Conclusions: Hepatocyte and MSC cotransplantation is feasible and safe in rat models of ALF. MSCs were found to survive the process and could be located within the periportal niches 2 weeks after treatment, without enhancing transplanted hepatocyte proliferation or differentiating into hepatocytes, while ameliorating the inflammatory response.

Keywords: Acute liver failure; Cell transplantation; Mesenchymal stem cells 


\section{HIGHLIGHTS}

- Hepatocyte and mesenchymal stem cell (MSC) cotransplantation was feasible and safe in rats of acute liver failure.

- Survived MSCs located within the periportal niches 2 weeks after treatment, without signs of enhancing transplanted hepatocyte proliferation or differentiating into hepatocytes but inflammation was ameliorated.

\section{INTRODUCTION}

Hepatocyte transplantation is a promising alternative to liver transplantation in patients with acute liver failure (ALF) and metabolic liver disease [1]. Hepatocyte transplantation is a safer and less invasive procedure for patients when compared to whole organ transplantation. Animal studies have clearly shown the efficacy of hepatocyte transplantation; however, this has not translated into clinical practice where there is a limited benefit [2]. Successful translation demands high-quality cell preparation, adequate cell numbers, viability, and efficient delivery [3]. In the case of ALF, the cell dose needed to rescue/reverse the outcome is far more than that for metabolic liver diseases [4]. Our results demonstrate that adequate rates of cellular infusion can increase engraftment of transplanted hepatocytes in a D-galactosamine-induced acute liver injury rat model [5]. However, the engraftment efficiency was only about $2.5 \% 1$ week after hepatocyte transplantation. In addition, it is difficult for hepatocytes to proliferate in the hostile microenvironment associated with ALF [6]. Considering the urgent need for large amounts of hepatocytes to treat ALF, there still much room for improvement in the cell based therapeutics.

In a retrorsine-treated liver injury rat model, immediate transplantation with either hepatocyte or bone marrow derived cells can ameliorate liver injury via a number of different mechanisms including hepatocyte proliferation in the former, and paracrine effects in the later [7]. Mesenchymal stem cells (MSCs), a major component of bone marrow derived cell mixtures, have been shown to exhibit multiple beneficial effects in vitro relevant to the liver injury therapeutic context, including (1) hepatocellular functional support (improved albumin secretion, ureagenesis, hepato-specific gene expression, cytochrome P450 activity) [8], (2) secretion of molecules that inhibit hepato- cyte apoptosis (such as stromal-cell-derived factor- 1 and vascular endothelial growth factor) [9-12] and stimulation of hepatocyte proliferation (via secretion of hepatocyte growth factor [HGF], epidermal growth factor, interleukin 6 [IL-6], and tumor necrosis factor- $\alpha$ [TNF- $\alpha$ ]), (3) modulation of the acute phase response and suppression of the inflammatory responses including IL-1 receptor antagonists and upregulation of anti-inflammatory cytokines like IL-10 [11], and finally (4) secrete several extracellular matrix molecules, including collagen, fibronectin and laminin necessary for liver reconstruction $[13,14]$. These observations suggest that MSC-derived cytokines could potentially protect the liver during injury.

In vivo, MSC or MSC-conditioned media can attenuate inflammation and augment cytokine and growth factor concentrations improving cell proliferation and providing an avenue for preventing fulminant hepatocyte failure [9,15-19]. MSC transplantation following solid organ transplantation, both clinically and experimentally, can also reduce the rate of acute rejection $[19,20]$. MSC transplantation alone, however, is not expected to exert any effect on AFL because the hostile microenvironment of ALF is not a good niche for MSCs and long-term engraftment rates are low [15]. Transplanted hepatocytes were unable to function, or even survive well, without stromal cell support. Thus, the addition of bone marrow-derived mesenchymal stromal cells (MSCs) during transplantation could support the proliferation and functionality of the transplanted hepatocytes [13].

There are over 280 registered clinical trials examining the application of MSC, 28 of which focus on the treatment of liver disease [15]. While, no severe side-effects have been reported so far, the long-term benefits of these treatments remain uncertain [15]. Li et al. [18] evaluated the transplantation of human bone-marrow-derived MSCs in a porcine model of acute liver failure (ALF induced with D-galactosamine) without the use of immunosuppressants. Most (13/15) achieved long-term survival $>6$ months) while all of the animals that did not have MSC treatment died [18]. Up to $30 \%$ of the hepatocytes, in this study, were shown to be derived from the bone marrow MSCs [18]. The underlying mechanisms for this response remain unknown, and are the subject of further investigation [16,21].

We have previously described the optimal rate for hepatocyte transplantation in an acute liver injury rat model to ensure optimal engraftment and repopulation [5]. If transplanted hepatocytes can proliferate properly, cell 
populations will double overtime improving the likelihood that there will be enough functional cells to compensate for the rapid loss of native hepatocytes and thus rescue the host's liver function. It is, therefore, reasonable to assume that cotransplantation of hepatocytes and MSCs could provide enough support to facilitate improved survival and proliferation of the transplanted hepatocytes, enhancing repopulation. The ability to properly repopulate the deteriorating liver is crucial in effective clinical intervention and thus the purpose of this study was to investigate the effects of MSC and hepatocyte cotransplantation in rats with acute liver injury.

\section{METHODS}

\section{Ethics Statement}

All animal experiments were approved by the Institutional Laboratory Animal Care and Use Committee of the $\mathrm{Na}$ tional University of Taiwan. All animals received humane care in accordance with the guidelines set out by the National Science Council of Taiwan (1997) and the Guide for the Care and Use of Laboratory Animals (National Institutes of Health publication 86-23, 1985 revision). All procedures were also performed in accordance with these guidelines.

\section{Animals}

Male Sprague-Dawley (SD) rats were used as recipient animals. Fluorescent SD rats (aged 8-10 weeks, 200-250 g) were purchased from the National Laboratory Animal Center in Taiwan and used as donor animals. These animals were bred in-house and maintained on standard laboratory chow and daily 12-hour light/dark cycles. All animal experiments were approved by the Institutional Laboratory Animal Care and Use Committee of the $\mathrm{Na}$ tional Taiwan University (No. 20130523 and 20150405).

\section{Isolation of Hepatocytes and MSCs for Transplantation}

In situ liver perfusion, collagenase digestion, and differential centrifugation were used to purify hepatocytes from GFP transgenic SD rats as previously described $[22,23]$. The viability and purity of each preparation were assessed using trypan blue exclusion on a hemocytometer. Isolated hepatocytes were resuspended $1 \times 10^{7}$ cells $/ \mathrm{mL}$ in phosphate buffered saline (PBS) without serum. Marrow cells, from both femurs and tibias were flushed from the bones of the DsRed transgenic SD rats using a syringe with a $26-G$ needle. Bone marrow mononuclear cells were isolated using Percoll gradient density centrifugation. MSCs were collected by depleting the cell suspensions of hematopoietic cells (CD45+) using anti-CD45 coated magnetic beads. Isolated MSCs were then resuspended to $1 \times 10^{6}$ cells $/ \mathrm{mL}$ in PBS without serum. MSCs were plated at a concentration of $10^{6}$ cells $/ \mathrm{mL}$ in murine MesenCult medium (Stemcell Technologies, Vancouver, Canada) and incubated at $37^{\circ} \mathrm{C}$ in a $5 \%$ humidified $\mathrm{CO}_{2}$ atmosphere for 3 hours and the unattached cells were then removed. Cells were put through a second round of purification if necessary [24].

\section{Hepatic Tissue Histology and the Determination of Liver Repopulation}

Fresh liver sections were fixed in formalin, embedded in paraffin, sectioned, and stained using hematoxylin and eosin to evaluate the histology of these samples. To identify transplanted hepatocytes in the recipient liver, DsRed expression was determined using fluorescence or enzyme histochemistry in liver cryosections. To analyze the liver repopulation, three to four sections from multiple liver lobes per rat were stained for DsRed activity. Microphotographs were obtained from consecutively adjacent areas to represent the whole section under $\times 100$ magnification using a digital camera. The relative occupation of these sections by the transplanted hepatocytes was evaluated and quantified using $\mathrm{J}$-Image software (National Cancer Institute, Bethesda, MD, USA).

\section{Experimental Design}

The D-galactosamine (Sigma, St. Louis, MO, USA) working solution was prepared as previously described [22] and used immediately after preparation. Acute hepatic injury was induced by D-galactosamine treatment $(0.9 \mathrm{~g} /$ $\mathrm{kg}$, intraperitoneal injection [IP]) in male SD rats 24 hours before transplantation. Isolated hepatocytes $\left(1 \times 10^{7} /\right.$ $\mathrm{mL}$ ) from $\mathrm{GFP}^{+} \mathrm{SD}$ rats were transplanted intraportally 24 hours after treatment with D-galactosamine at an infusion rate of 70 seconds. DsRed ${ }^{+}$MSCs $(0.5,1$, or $2 \times 10^{6} / 0.5 \mathrm{~mL}$ ) were transplanted intraportally 1 hour after hepatocyte transplantation. The surviving rats were sacrificed and their livers harvested at 1,2, and 4 weeks after transplantation (Fig. 1A).

\section{Liver Tissue Evaluations}

All immunofluorescent/immunohistochemical staining 
A
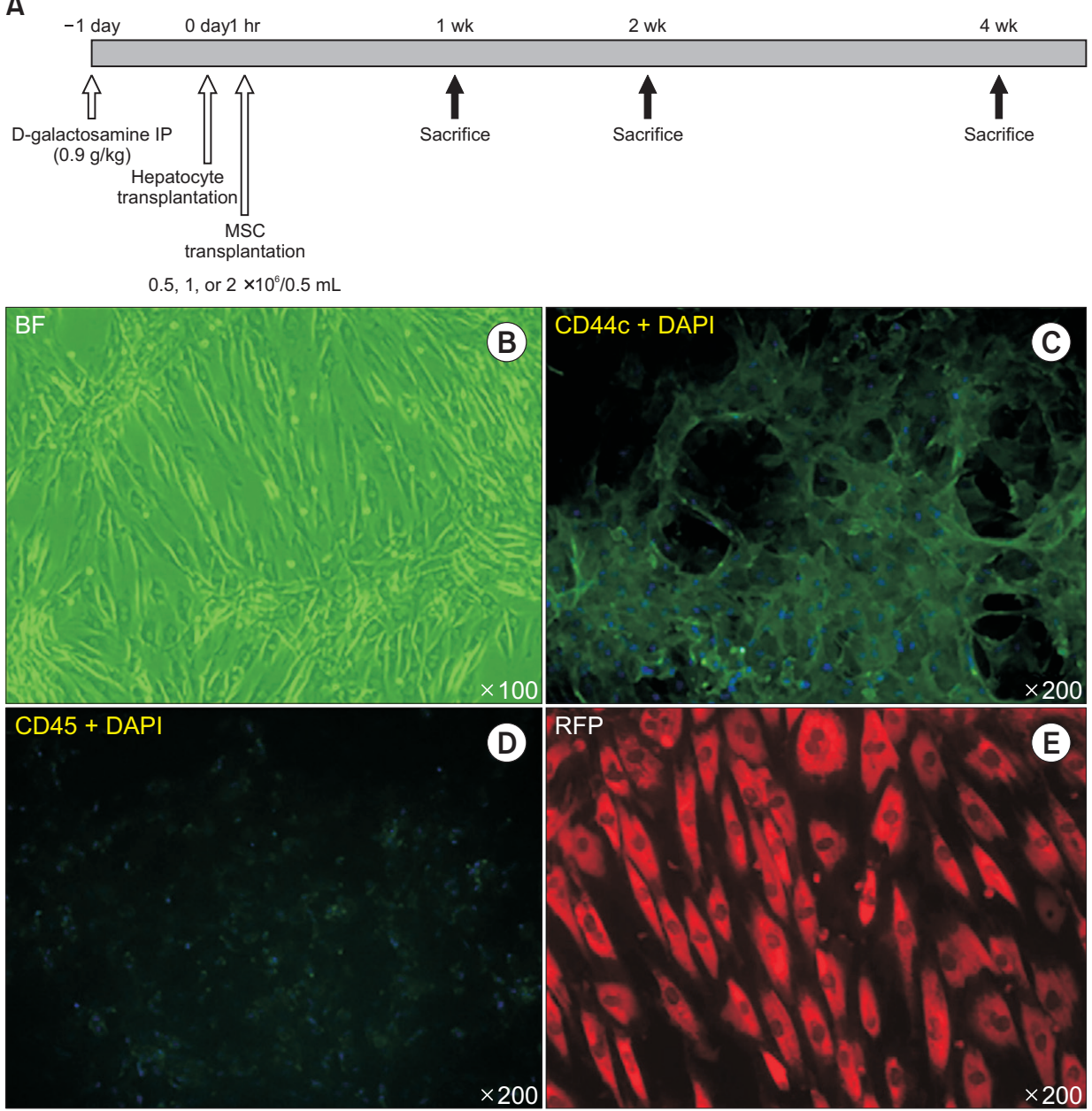

Fig. 1. Model of acute liver failure (ALF) used in the cotransplantation study. (A) Experimental design for the cotransplantation of rat hepatocytes and mesenchymal stem cells (MSCs) in a rat model of ALF. (B-E) Characterization of MSC. The MSCs were spindle shaped (B) and expressed CD44C (C) but not CD45 (D). They were fluoro-red $(+)(E)$ since they were derived from bone marrow aspirates of DsRed transgenic Sprague-Dawley rats. IP, intraperitoneal injection; BF, blank field; DAPI, 4', 6-diamidino-2-phenylindole; RFP, red fluorescent protein.

was performed according to previously described protocols [25]. Quantitative Reverse Transcriptase polymerase chain reaction was used to evaluate tissue specific expression of HGF, EGF, SCF, IL-6, IL-10, TNF $\alpha, T G F \beta 1$, and collagen I and was performed as previously [25].

\section{Serological Assay}

Hepatic venous blood was sampled after the recipient rats were sacrificed. Biochemical analyses (aspartate aminotransferase [AST], alanine aminotransferase [ALT], alkaline phosphatase [ALP], lactate dehydrogenase [LDH], ammonia, albumin, and blood urea nitrogen [BUN]) were performed in an animal laboratory using the standard automated assays, as previously described [5].

\section{Statistical Analysis}

At least four animals per treatment were evaluated. Data are shown in a qualitative manner or presented as the mean, as appropriate. No animal data were excluded.

\section{RESULTS}

\section{Characterization of Donor MSCs}

MSCs in culture before transplantation are shown in Fig. 1B-E. They maintained the classic spindle-shape morphologically (Fig. 1B), expressed CD44 (Fig. 1C) and did not express hematopoietic cell marker CD45 (Fig. 1D). They were derived from the bone marrow aspirations from the DsRed SD rats and were therefore fluoro-red positive.

\section{Histopathological Changes after Hepatocyte and MSC Co- transplantation}

One day after D-gal administration, livers of SD rats showed extensive hepatocyte necrosis, periportal focal 


\section{A}

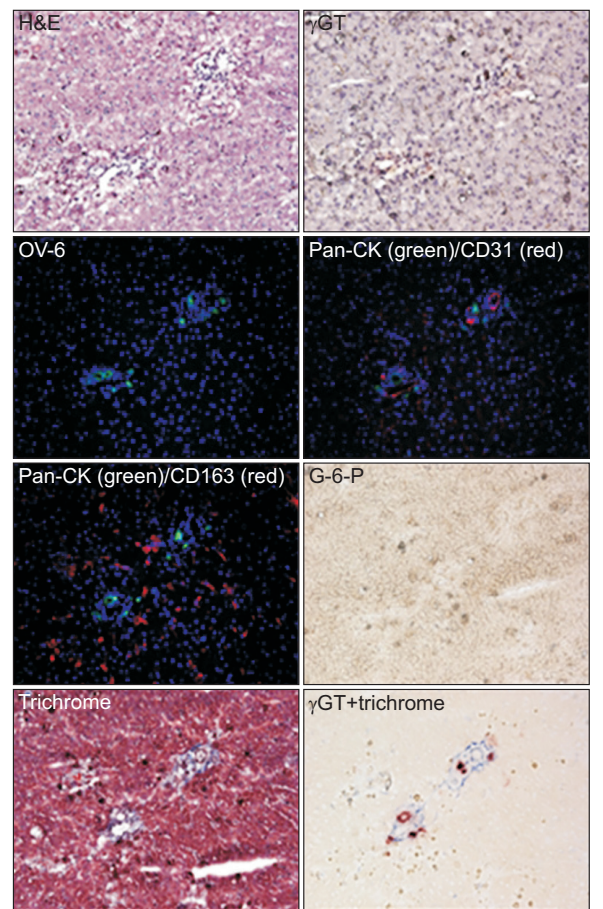

B $\quad H \& E$

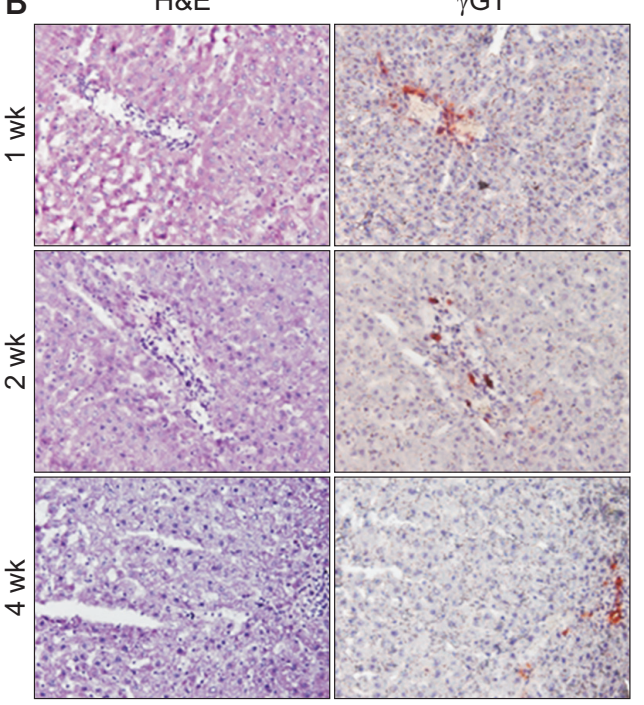

C

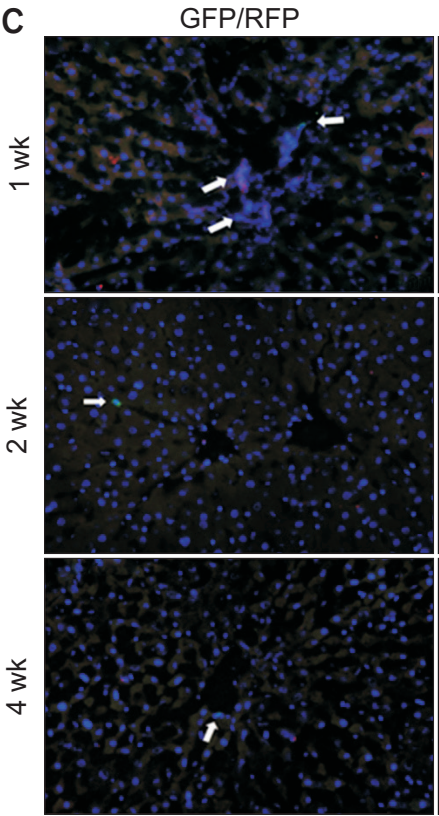

CD31/RFP

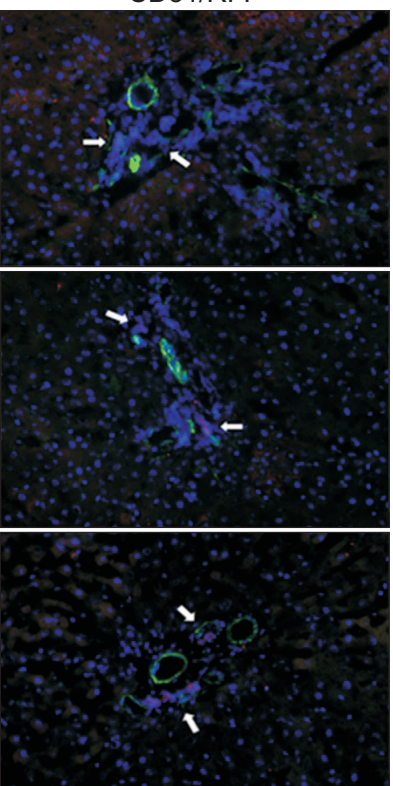

CD163
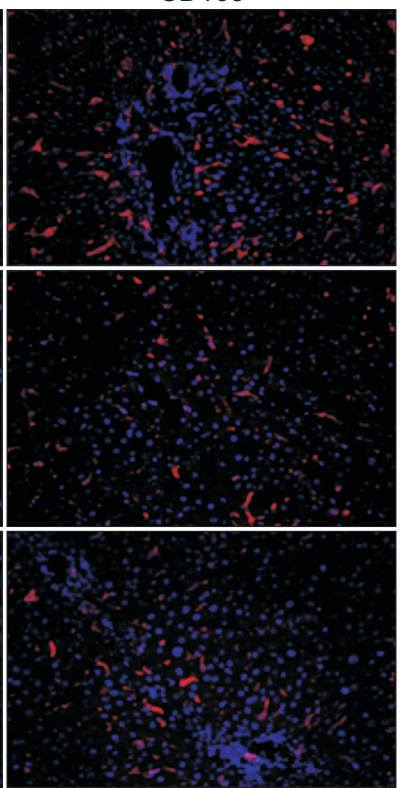

Fig. 2. Histopathological changes after hepatocyte and mesenchymal stem cell (MSC) cotransplantation in acute liver injury. (A) Control liver. Acute liver injury developed, 1 day after D-galactosamine treatment. Ductular reactions were noted by oval cell marker (OV-6), expression near the portal vein. Prominent $\mathrm{CD}_{163^{+}}$macrophage and low G-6-P expression were also observed. Fibrosis was limited to the periportal region. (B) One, 2 and 4 weeks after cotransplantation, $\gamma$-glutamyltransferase $(\gamma \mathrm{GT})$, was expressed in the ductular cells along with obvious signs of liver recovery. (C) Tracing of transplanted donor cells after cotransplantation. Donor hepatocytes were labelled green and MSCs were labelled red. CD163 marker expression was used to determine macrophage infiltration after cotransplantation and is shown on the right. Arrows indicate the donor cells. CD31 marks the endothelial inner lining of the portal vein. GFP, green fluorescent protein; RFP, red fluorescent protein. Magnification, all $\times 200$. 
A

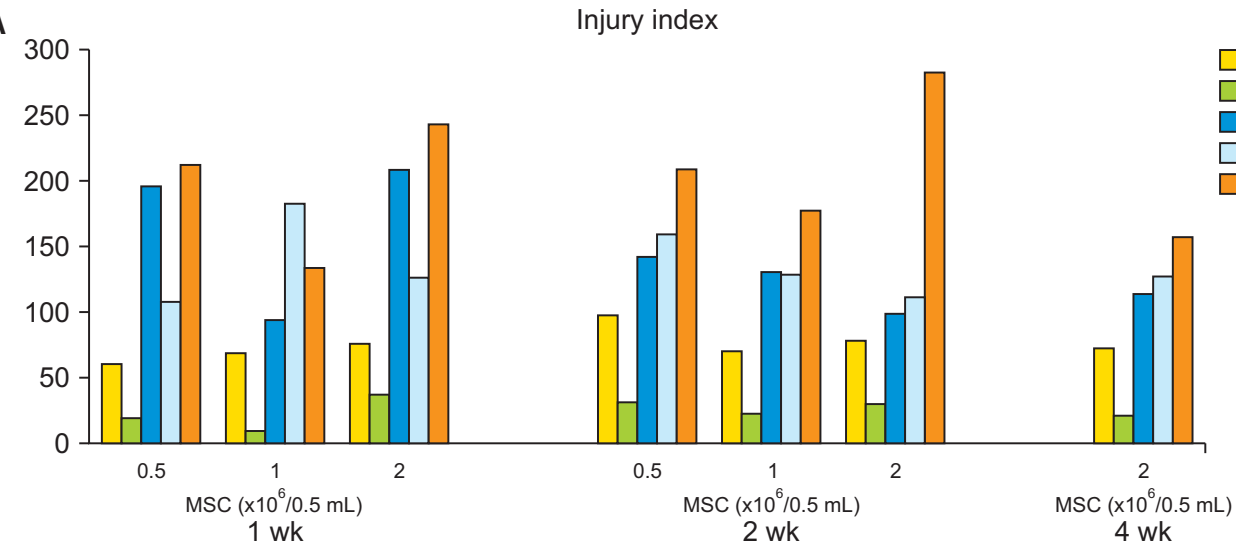

AST (U/L)

ALT $(U / L)$

$\operatorname{ALP}(\mathrm{U} / \mathrm{L})$

LDH (U/L)

Ammonia level (umol/L)
B

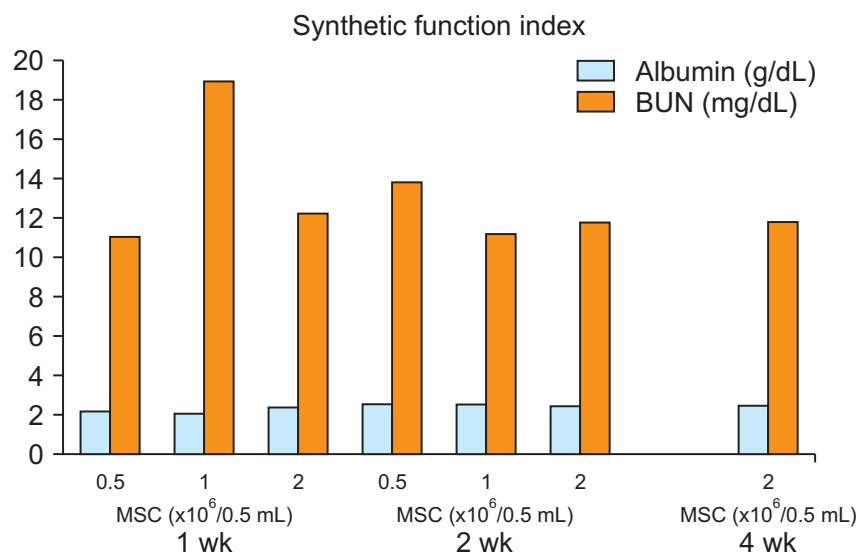

C

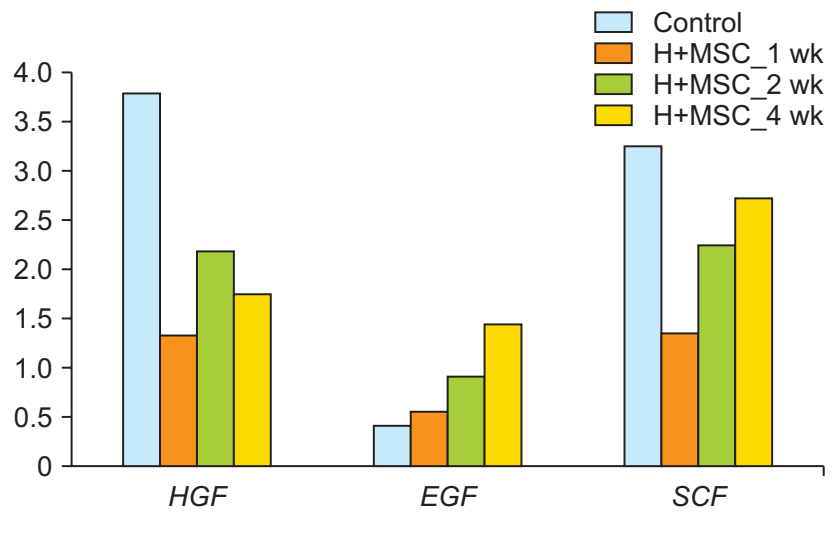

D

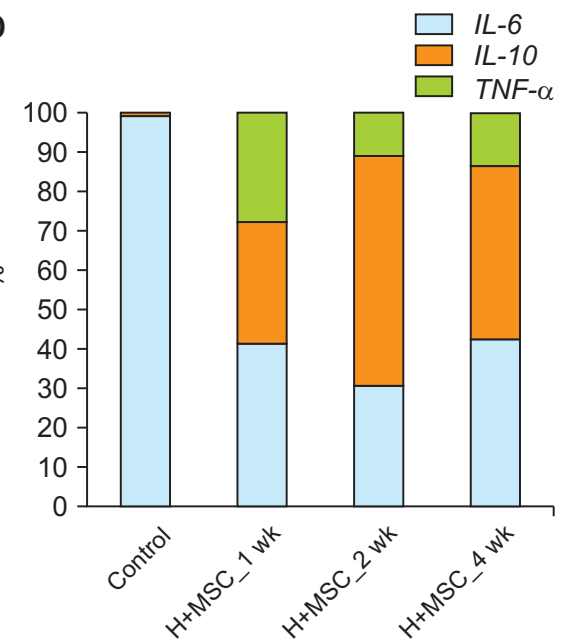

E

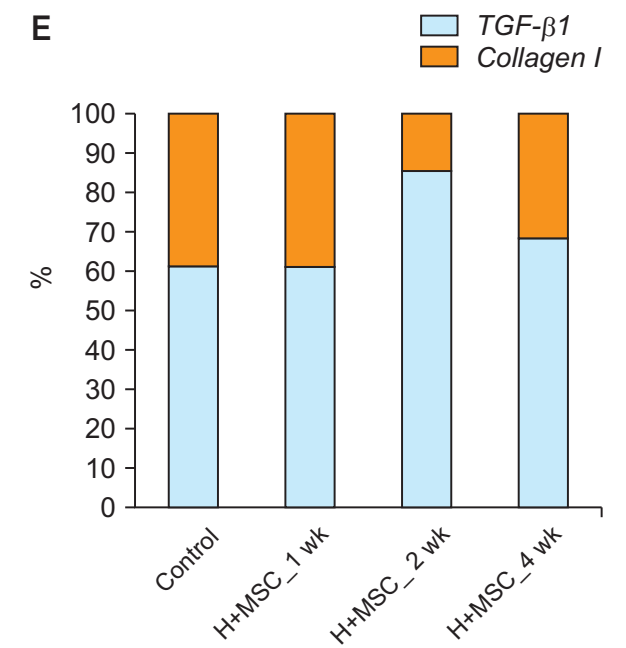

TGF- $\beta 1$

Collagen I

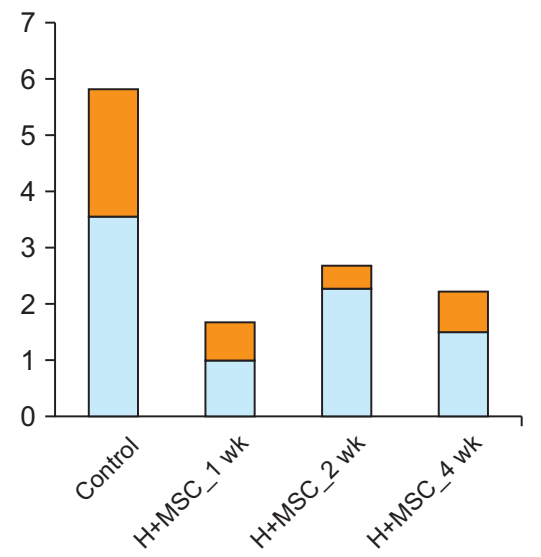

Fig. 3. Serological marker and gene expression changes. Serological changes after cotransplantation of hepatocyte and mesenchymal stem cells (MSCs) in rats with acute liver injury. Markers of injury index (A) and synthetic function index (B). Gene expression of growth factors (C), inflammation (D) and fibrosis (E) after cotransplantation. Control: acute liver injury 1 day after D-galactosamine treatment; Baseline expression: glyceraldehyde 3-phosphate dehydrogenase; AST, aspartate aminotransferase; ALT, alanine aminotransferase; ALP, alkaline phosphatase; LDH, lactate dehydrogenase; BUN, blood urea nitrogen; $H$, hepatocyte; HGF, hepatocyte growth factor; EGF, epidermal growth factor; SCF, stem cell factor; IL, interleukin; TNF- $\alpha$, tumor necrosis factor- $\alpha$; TGF- $\beta 1$, transforming growth factor- $\beta 1$. 
expression of $\gamma$-glutamyltransferase, oval cell marker (OV-6), and pan-cytokeratin. No fibrosis was evident at this stage. Multiple $\mathrm{CD}_{163^{+}}$macrophages were present in the parenchyma sparing the portal area (Fig. 2A). After cotransplantation, the liver parenchyma was seen to undergo progressive restoration with reduced inflammatory cell infiltration (Fig. 2B). However, biliary ductular proliferation can still be observed 4 weeks after cotransplantation.

\section{Tracing the Origins of the Transplanted Hepatocytes and MSCs}

Transplanted MSCs (fluoro-red) were located within the periportal area, 1, 2, and 4 weeks after cotransplantation (Fig. 2C). Transplanted hepatocytes (fluoro-green) were found scattered throughout the parenchyma but did not show any signs of proliferation after engraftment $(1,2$, and 4 weeks) (Fig. 2C). Transplanted MSCs did not appear to differentiate into hepatocytes or proliferate during this study period.

\section{Serological Changes and Synthetic Functional Recovery Following Hepatocyte and MSC Transplantation: Comparing Different Doses of MSCs}

After cotransplantation, we evaluated changes in the serological markers for liver injury (AST, ALT, ALP, LDH; indicating liver injury) and synthetic markers (albumin, BUN) with the results shown in Fig. $3 A$ and B, respectively. When we compared the serological results after 1 and 2 weeks of cotransplantation with MSCs, at doses of 0.5 or $2 \times 10^{6} / 0.5 \mathrm{~mL}$, we were able to determine that $1 \times 10^{6} \mathrm{MSCs}$ was the correct dose for future cotransplantation studies.

\section{Gene Expression in Liver Tissues Following Cotransplantation}

Gene expression profiles of genes associated with growth factors, inflammation-related cytokines, and fibrosis were evaluated at 1, 2, and 4 weeks after cotransplantation (Fig. 3C-E). HGF and SCF were highly expressed in the D-galactosamine induced acute liver injury (control) samples. After cotransplantation, EGF and SCF progressively increased over the 4-week follow-up period (Fig. 3C). In addition, IL-10 expression increased, and expression of both IL-6 and TNF- $\alpha$, progressively decreased, within the first 2 weeks following cotransplantation (Fig. 3D). The gene expression profiles for TGF- $\beta 1$ and Collagen I, associated with fibrosis, remained similar in the first week after cotransplantation, after which they decreased and then fluctuated for the rest of the study period (Fig. 3E).

$\mathrm{CD}_{163^{+}}$Macrophages after Cotransplantation in D-GalInduced Acute Liver Injury Rats

When we compared CD163 expression at 1 week after cotransplantation, we observed a reduction in the proportion of cytoplasm and $\mathrm{CD}_{163^{+}}$macrophages at both 2 and 4 weeks after cotransplantation (Fig. 2C).

\section{DISCUSSION}

This study made four key observations. MSC cotransplantation did not directly benefit donor hepatocyte proliferation. Transplanted MSCs, mostly resided in the periportal niche, and did not differentiate into liver cells. IL-10 gene expression, the anti-inflammatory cytokine, increased proportionally 1 week after cotransplantation. While, $\mathrm{CD}_{163^{+}}$macrophages were prominent with large amounts of cytoplasm.

Transplanted MSCs did not differentiate into liver cells in our study. Although other studies have demonstrated that MSCs can differentiate into cells from the terminal organs in vivo, there is growing scepticism about their functional differentiation beyond their differentiation to adipocytes, osteocytes and chondrocytes [26,27]. Current rationales have shifted toward an emphasis on the paracrine effects and immunomodulation of MSC cell therapies within the tissue microenvironment $[26,28]$. Wang et al. [27] showed that IL-10 secreted by MSCs attenuated ALF by inhibiting pyroptosis in mice. Our study showed significant changes in the cytokine profile of liver tissues 1 and 2 weeks after cotransplantation with a definite decrease in the expression of the inflammatory cytokines. $I L-10$ gene expression increased in direct proportion to IL-6 expression after cotransplantation. Whether these induced mRNAs are derived from the transplanted MSCs needs further investigation.

ALF is characterized by sequential and overlapping episodes of hepatocyte death-related inflammation, followed by the induction of the anti-inflammatory response with or without resultant immune paralysis and sepsis, followed by liver repair and recovery, if the host survives [29]. In our ALF model, the gene expression of IL-6 was very high, one day after $\mathrm{D}$-galactosamine treatment (data not shown). Meanwhile, IL-10 was elevated and CD163 macrophage appeared in the liver microenvironment 
shortly thereafter. At 2 weeks after cotransplantation, although the proportion of $I L-10$ increased, this expression gradually decreased compared to the initial values. This observation suggests that the microenvironment settled down soon after cotransplantation. Consistently, gene expression associated with inflammation-related cytokines was reduced 1 week after cotransplantation when we compared MSC cotransplantation and hepatocyte-only transplantation (data not shown).

Clinical challenges of MSC application include variabilities among the large number of disease categories, different routes of delivery, range of doses, and types of MSCs being used [30]. The low success rate for meeting primary outcomes in clinical trials actually underscores the need for new designs [30]. Our study provides a reasonable expectation for potential human effect of MSC cotransplantation (reducing micro-environmental inflammation of ALF) that could aid in setting reasonable outcomes for future trial designs (such as inflammation score).

This study has one major limitation, the data generated in this study was insufficient to generate an interactive mechanism for the MSCs and the microenvironment, which is an inherent issue with rat studies of this nature. In summary, hepatocyte and MSC cotransplantation is feasible and safe in an acute liver injury rat model. Transplanted MSCs were located within the periportal niches up to 4 weeks after transplantation without any signs of enhancing transplanted hepatocyte proliferation or their differentiation into hepatocytes themselves. Inflammation was ameliorated by cotransplantation.

\section{ACKNOWLEDGMENTS}

\section{Conflict of Interest}

No potential conflict of interest relevant to this article was reported.

\section{Funding/Support}

This study was funded by the Ministry of Science and Technology, Taiwan (MOST103-2314-B-002-125-MY2, 105-2314-B-002-159).

\section{Previous Presentation}

Part of this work has been presented at the International Digestive Forum in 2016.

\section{ORCID}

Cheng-Maw Ho https://orcid.org/0000-0003-1874-7615

Hui-Ling Chen https://orcid.org/0000-0002-2572-0595

Rey-Heng Hu https://orcid.org/0000-0001-6709-031X

Po-Huang Lee https://orcid.org/0000-0001-5831-035X

\section{Author Contributions}

Conceptualization: $\mathrm{CMH}, \mathrm{HLC}$. Data curation: $\mathrm{CMH}, \mathrm{YHC}$, CSC, HLC. Formal analysis: CMH, HLC, RHH, PHL. Funding acquisition: $\mathrm{CMH}, \mathrm{HLC}, \mathrm{PHL}$. Methodology: $\mathrm{CMH}, \mathrm{YHC}$, SLH, HLC. Project administration: CSC, RHH. Visualization: all author. Writing-original draft: $\mathrm{CMH}$. Writing-review \& editing: $\mathrm{CMH}, \mathrm{HLC}$.

\section{REFERENCES}

1. Iansante V, Mitry RR, Filippi C, Fitzpatrick E, Dhawan A. Human hepatocyte transplantation for liver disease: current status and future perspectives. Pediatr Res 2018;83:232-40.

2. Soltys KA, Soto-Gutiérrez A, Nagaya M, Baskin KM, Deutsch $M$, Ito R, et al. Barriers to the successful treatment of liver disease by hepatocyte transplantation. J Hepatol 2010;53:769-74.

3. Donato MT, Lahoz A, Montero S, Bonora A, Pareja E, Mir J, et al. Functional assessment of the quality of human hepatocyte preparations for cell transplantation. Cell Transplant 2008;17:1211-9.

4. Strom SC, Chowdhury JR, Fox IJ. Hepatocyte transplantation for the treatment of human disease. Semin Liver Dis 1999;19:39-48.

5. Ho CM, Chen YH, Chien CS, Ho YT, Ho SL, Hu RH, et al. Transplantation speed offers early hepatocyte engraftment in acute liver injured rats: a translational study with clinical implications. Liver Transpl 2015;21:652-61.

6. Riordan SM, Williams R. Acute liver failure: targeted artificial and hepatocyte-based support of liver regeneration and reversal of multiorgan failure. $\mathrm{J}$ Hepatol 2000;32(1 Suppl):63-76.

7. Zhang B, Inagaki M, Jiang B, Miyakoshi M, Arikura J, Ogawa $\mathrm{K}$, et al. Effects of bone marrow and hepatocyte transplantation on liver injury. J Surg Res 2009;157:71-80.

8. Gómez-Aristizábal A, $\mathrm{Ng} \mathrm{C}, \mathrm{Ng}$ J, Davies JE. Effects of two mesenchymal cell populations on hepatocytes and lymphocytes. Liver Transpl 2012;18:1384-94. 
9. van Poll D, Parekkadan B, Cho CH, Berthiaume F, Nahmias $Y$, Tilles AW, et al. Mesenchymal stem cell-derived molecules directly modulate hepatocellular death and regeneration in vitro and in vivo. Hepatology 2008;47:1634-43.

10. Balber AE. Concise review: aldehyde dehydrogenase bright stem and progenitor cell populations from normal tissues: characteristics, activities, and emerging uses in regenerative medicine. Stem Cells 2011;29:570-5.

11. Ortiz LA, Dutreil M, Fattman C, Pandey AC, Torres G, Go K, et al. Interleukin 1 receptor antagonist mediates the antiinflammatory and antifibrotic effect of mesenchymal stem cells during lung injury. Proc Natl Acad Sci U S A 2007;104:11002-7.

12. Hematti P. Role of mesenchymal stromal cells in solid organ transplantation. Transplant Rev (Orlando) 2008;22:262-73.

13. Gómez-Aristizábal A, Keating A, Davies JE. Mesenchymal stromal cells as supportive cells for hepatocytes. Mol Ther 2009;17:1504-8.

14. Yagi $H$, Parekkadan B, Suganuma K, Soto-Gutierrez A, Tompkins RG, Tilles AW, et al. Long-term superior performance of a stem cell/hepatocyte device for the treatment of acute liver failure. Tissue Eng Part A 2009;15:3377-88.

15. Meier RP, Müller YD, Morel P, Gonelle-Gispert C, Bühler LH. Transplantation of mesenchymal stem cells for the treatment of liver diseases, is there enough evidence? Stem Cell Res 2013;11:1348-64.

16. Liu WH, Song FQ, Ren LN, Guo WQ, Wang T, Feng YX, et al. The multiple functional roles of mesenchymal stem cells in participating in treating liver diseases. $J$ Cell Mol Med 2015;19:511-20.

17. Kanazawa H, Fujimoto $Y$, Teratani T, Iwasaki J, Kasahara $\mathrm{N}$, Negishi $\mathrm{K}$, et al. Bone marrow-derived mesenchymal stem cells ameliorate hepatic ischemia reperfusion injury in a rat model. PLoS One 2011;6:e19195.

18. Li J, Zhang L, Xin J, Jiang L, Li J, Zhang T, et al. Immediate intraportal transplantation of human bone marrow mesenchymal stem cells prevents death from fulminant hepatic failure in pigs. Hepatology 2012;56:1044-52.

19. Tan J, Wu W, Xu X, Liao L, Zheng F, Messinger S, et al. Induction therapy with autologous mesenchymal stem cells in living-related kidney transplants: a randomized controlled trial. JAMA 2012;307:1169-77.
20. Wan CD, Cheng R, Wang HB, Liu T. Immunomodulatory effects of mesenchymal stem cells derived from adipose tissues in a rat orthotopic liver transplantation model. Hepatobiliary Pancreat Dis Int 2008;7:29-33.

21. Baertschiger RM, Serre-Beinier V, Morel P, Bosco D, Peyrou M, Clément $S$, et al. Fibrogenic potential of human multipotent mesenchymal stromal cells in injured liver. PLoS One 2009;4:e6657.

22. $\mathrm{Yu} \mathrm{CH}$, Chen $\mathrm{HL}$, Chen WT, Ni YH, Lin YL, Chang MH. Portal hemodynamic changes after hepatocyte transplantation in acute hepatic failure. J Biomed Sci 2004;11:756-63.

23. Rajvanshi P, Kerr A, Bhargava KK, Burk RD, Gupta S. Studies of liver repopulation using the dipeptidyl peptidase IV-deficient rat and other rodent recipients: cell size and structure relationships regulate capacity for increased transplanted hepatocyte mass in the liver lobule. Hepatology 1996;23:482-96.

24. Soleimani M, Nadri S. A protocol for isolation and culture of mesenchymal stem cells from mouse bone marrow. Nat Protoc 2009;4:102-6.

25. Yu CH, Chen HL, Chen YH, Chang MF, Chien CS, Chang $\mathrm{MH}$. Impaired hepatocyte regeneration in acute severe hepatic injury enhances effective repopulation by transplanted hepatocytes. Cell Transplant 2009;18:1081-92.

26. Schäfer R, Spohn G, Baer PC. Mesenchymal stem/ stromal cells in regenerative medicine: can preconditioning strategies improve therapeutic efficacy? Transfus Med Hemother 2016;43:256-67.

27. Wang J, Ren H, Yuan X, Ma H, Shi X, Ding Y. Interleukin-10 secreted by mesenchymal stem cells attenuates acute liver failure through inhibiting pyroptosis. Hepatol Res 2018;48:E194-202.

28. Lee SC, Kim KH, Kim OH, Lee SK, Hong HE, Won SS, et al. Determination of optimized oxygen partial pressure to maximize the liver regenerative potential of the secretome obtained from adipose-derived stem cells. Stem Cell Res Ther 2017;8:181.

29. Possamai LA, Thursz MR, Wendon JA, Antoniades CG. Modulation of monocyte/macrophage function: a therapeutic strategy in the treatment of acute liver failure. J Hepatol 2014;61:439-45.

30. Galipeau J, Sensébé L. Mesenchymal stromal cells: clinical challenges and therapeutic opportunities. Cell Stem Cell 2018;22:824-33. 